\title{
GANANCIA GENÉTICA ESPERADA EN LA SELECCIÓN DE ACACIA (Acacia mangium WILLD.) EN CÓRDOBA (COLOMBIA)
}

\section{EXPECTED GENETIC GAIN IN THE SELECTION OF ACACIA (Acacia mangium WILLD.) IN CORDOBA (COLOMBIA)}

\author{
Miguel Espitia', Olman Murillo², Carlos Castillo³, Hermes Araméndiz, Nelson Paternina ${ }^{5}$
}

\begin{abstract}
${ }^{1}$ Docente Investigador Universidad de Córdoba, Ph.D. mespitia@sinu.unicordoba.edu.co. Diagonal 50 No. 8-105, Barrio Villa del Río, Montería (Córdoba). ${ }^{2}$ Profesor Titular Instituto Tecnológico de Costa Rica, Ph.D. olmuga@yahoo.es. ${ }^{3}$ Ingeniero Agrónomo Universidad de Córdoba. carloscastillo1232@hotmail.com. ${ }^{4}$ Docente Investigador Universidad de Córdoba, Ph.D. haramendiz@hotmail.com. ${ }^{5}$ Tecnólogo Forestal 3F Kanguroid. napaternina@hotmail.com
\end{abstract}

Rev. U.D.C.A Act. E Div. Cient. 13 (2): 99-107, 2010

\section{RESUMEN}

La agenda de competitividad de Córdoba (Colombia), incluye la plantación de 200 mil hectáreas de forestales, para el 2025, con especies de alta demanda en el mercado de madera internacional. La acacia (Acacia mangium Willd.) ha sido recomendada por su excelente adaptación y crecimiento. Se estimó la ganancia genética esperada (GG) en la selección de árboles plus, en 3.626 ha de acacia, para el diámetro a la altura del pecho (DAP), altura comercial (ALCO), volumen comercial (VOLCO) y calidad del fuste (CALI). La selección, se basó en la evaluación fenotípica del árbol candidato y sus cuatro mejores vecinos, en un radio de $20 \mathrm{~m}$. Los árboles seleccionados, se clasificaron en lista $\mathrm{A}$, cuando registraron superioridad en volumen y en calidad, con base en el diferencial de selección (S) y, en lista B, cuando superaron solamente en volumen o en calidad a todos sus vecinos. La $\mathrm{GG}$, se estimó por GG $=\mathrm{S}^{*} \mathrm{~h}^{2}$, donde $\mathrm{h}^{2}$ es la heredabilidad en sentido estrecho promedio reportada. Se determinó un Índice de Selección (IS), que integró, de forma ponderada, el VOLCO, con CALI. De 89 árboles seleccionados, 32 fueron clasificados como plus $\mathrm{A}$, con valores de diferencial de selección de $22 \%, 89 \%, 194 \%$ y $52 \%$, para DAP, ALCO, VOLCO y CALI, respectivamente. Al seleccionar los 15 mejores árboles $A$, con base en el IS, se espera obtener ganancias genéticas de 4,38\%; 29,76\%; 61,75\% y 27,27\%, para DAP, ALCO, VOLCO y CALI, respectivamente. Los resultados sugieren un progreso genético importante en el mejoramiento genético de acacia en Córdoba.

Palabras clave: Árboles plus, diferencial de selección, volumen comercial, calidad, índice de selección.

\section{ABSTRACT}

The competitiveness strategy for Cordoba (Colombia) includes the planting of 200 thousand hectares of forest with species of high demand in the international wood market by the year 2025. The Acacia mangium (Willd) has been recommended because of its excellent adaptation and growth. The expected genetic gain (GG) in the selection of plus trees of acacia in 3626 hectares for the diameter at breast height (DAP), commercial height (ALCO), trade volume (VOLCO) and log quality (CALI) was estimated. The selection was based on the phenotypic evaluation of the candidate tree and its four best neighbors within a radius of $20 \mathrm{~m}$. The selected trees were classified in list A when superiority, based on the selection differential (S), in volume and quality was recorded and in list $B$, when they only exceeded in either volume or quality all their neighbors. The GG was estimated by GG $=S^{*} h^{2}$, where $h^{2}$ is the narrow sense heritability average reported. A Selection Index (SI) was determined, that integrated in weigh up form VOLCO and CALI. From 89 selected trees, 32 were classified as plus $A$, with values of selection differential of $22 \%$ (DAP), of $89 \%$ (ALCO), of $194 \%$ (VOLCO) and of 52\% (CALI). In selecting of the 15 best A trees based on IS, a genetic gain of $4.38 \%$ (DAP), of $29.76 \%$ (ALCO), of $61.75 \%$ (VOLCO) and of $27.27 \%$ (CALI) is expected. The results suggest an important genetic progress in the breeding of acacia in Cordoba.

Key words: Plus trees, selection differential, commercial volume, quality, selection index. 


\section{INTRODUCCIÓN}

Colombia cuenta con 25 millones de hectáreas, aproximadamente, con aptitud forestal, de las cuales, el departamento de Córdoba posee 897.086ha (Rincón, 2009; MADR, 2005). En el departamento de Córdoba existen 15.000ha de plantaciones forestales (nativas e introducidas) y en los próximos 25 años, se espera plantar 200.000ha, en las principales zonas productoras (CFC, 2000). Entre las especies introducidas plantadas para la producción de madera, con fines comerciales, se encuentran teca (Tectona grandis L. F), melina (Gmelina arborea Roxb.) y acacia (A. mangium Willd.), que proveen madera de alta calidad y de demanda en el mercado internacional.

La acacia es una especie perteneciente a la familia Leguminosae, nativa del noroeste de Australia, del este de Indonesia, incluyendo las islas Molucas y Papúa-Nueva Guinea (CATIE, 1992). El árbol alcanza de 25 a 30m de altura y el DAP puede ser superior a los $35 \mathrm{~cm}$; crece en cualquier tipo de suelo y su madera es calificada semidura, resistente y tiene múltiples usos (Trujillo, 2007). Según Ladrach (2010), la $A$. mangium es una de las especies más utilizadas en el mundo para producción de pulpa, junto con varias especies e híbridos clonales de Eucalyptus spp. y Pinus spp.

La actividad forestal ha aumentado, significativamente, como consecuencia de la escasez de madera del bosque natural y la creciente demanda de productos forestales, por parte de la población; sin embargo, para convertir la actividad forestal en un proceso productivo rentable y seguro es necesario desarrollar programas de mejoramiento y de manejo, que conduzcan a la obtención de materia prima de la más alta calidad, con el menor costo posible (Murillo E Badilla, 2004).

El desarrollo de las tecnologías de propagación in vivo han permitido un progreso asombroso en el cultivo de eucaliptos en el mundo (Xavier et al. 2009), donde la productividad avanzó de $200 \mathrm{~m}^{3}$ ha $^{-1}$ en los años 70 's, a superar, actualmente, la barrera de los $400 \mathrm{~m}^{3} \mathrm{ha}^{-1}$, a nivel operativo, con resultados de investigación que superan los $500 \mathrm{~m}^{3} \mathrm{ha}^{-1}$, en siete años. Estos resultados son el producto de la visión de las plantaciones como un cultivo, donde confluyen tres componentes vitales: Suelo + Semilla + Manejo (Murillo \& Badilla, 2005).

Los principales problemas para la producción forestal en el país y en el departamento, específicamente, con A. mangium, radican en: a) bajo rendimiento; b) escasez de semilla (sexual o asexual) como material base para atender la demanda de siembra; c) dificultad para importar semilla (sexual o asexual) mejorada y, d) ausencia de un programa de mejoramiento genético sexual y clonal (CFC, 2000).
El éxito de un programa de mejoramiento genético (PMG) depende de la calidad e intensidad de selección (rigor) de los árboles parentales. Las ganancias esperadas derivan, tanto del control genético de las características de interés como de la variabilidad existente en la población (Zobel $\&$ Talbert, 1988; Balcorta $\&$ Vargas, 2004). La heredabilidad en sentido estricto y el diferencial de selección son útiles para predecir la respuesta de la selección en especies forestales (Zobel $\varepsilon$ Talbert, 1988). El diferencial de selección es importante, porque está altamente correlacionado con la ganancia genética, que es el fin de un programa de mejoramiento genético (Balcorta \& Vargas, 2004).

De manera general, se ha utilizado el diferencial de selección fenotípico, obtenido durante la selección de los árboles plus (árboles superiores en volumen, en calidad y en sanidad a sus mejores vecinos), como base para estimar el progreso genético esperado en teca (Vallejos et al. 2010). Las estimaciones de ganancia genética esperada, le permiten al mejorador forestal conocer su progreso genético potencial y decidir, al inicio del programa, cuáles individuos componen la población comercial y cuáles la población de mejoramiento (Vallejos et al. 2010).

Existen diversos reportes de varios países sobre intensidad de selección (i) y ganancia genética (GG), para varios caracteres de interés en programas jóvenes de mejoramiento genético forestal, donde se reportan valores que oscilan desde " $i$ ", de un árbol plus cada 3.000 a 25.000 árboles y ganancias genéticas entre un 15 a un $25 \%$ en volumen (Vallejos et al. 2010; Rocha et al. 2009; Murillo \& Badilla, 2009; Verryn et al. 2009; Blada \& Popescu, 2008; Botrel et al. 2007; Han et al. 2007; Rojas \& Arias, 2004; Kumar \& Matharoo, 2003; Cornelius, 1994; Cornelius $\&$ Hernández, 1994), pero no se encontraron artículos que referencian a A. mangium.

El objetivo de este trabajo fue realizar un proceso de selección de árboles plus en plantaciones comerciales de acacia ( $A$. mangium Willd.), estimar su diferencial de selección y la ganancia genética esperada, con el propósito de iniciar un programa de mejoramiento genético, para las condiciones forestales del Departamento de Córdoba (Colombia).

\section{MATERIALES Y MÉTODOS}

El estudio, se realizó durante el 2008 y 2009, en nueve plantaciones de acacia (A. mangium Willd.), del departamento de Córdoba (Colombia), de siete o más años de edad, las cuales, sumaron un total de 3.626 hectáreas (Tabla 1). La selección, se efectuó a través de un barrido exhaustivo de toda el área plantada; el proceso de selección de los árboles plus, mediante visita y participación de trabajadores locales de las plantaciones, quienes fueron entrenados, utilizando la metodología propuesta por Zobel \& Talbert (1984), refinada 
y mejorada en la cooperativa de mejoramiento forestal GENFORES (Vallejos et al. 2010). Esta metodología ha sido empleada y mejorada en Costa Rica, en los últimos diez años, y supera las limitaciones de las metodologías tradicionales, cuando existen diferencias en edad y en calidad de sitio, entre los árboles seleccionados e, incorpora, la calidad del fuste y un índice de selección, como nuevos caracteres de mayor impacto económico (Murillo et al. 2004). Todos los árboles pre-seleccionados y sancionados en cada finca fueron georeferenciados, para poder colectar su semilla y sus brotes, posteriormente.
La selección, se basó en la evaluación fenotípica del árbol candidato y sus cuatro mejores vecinos, en un radio de $20 \mathrm{~m}$, considerando los siguientes caracteres deseables: a) árbol sin gambas; b) fuste rectilíneo (FUSTE); c) pocos nudos; d) copa del árbol pequeña y simétrica; e) ángulo de inserción de ramas de 45 a $90^{\circ}$ y delgadas (RAMA); f) dominante en altura; g) sanidad del árbol (SANIDAD); h) diámetro a la altura del pecho (DAP); i) altura comercial (ALCO); j) volumen comercial (VOLCO) y, k) calidad del fuste (CALI). El VOLCO, se estimó aplicando la fórmula conicidad, que incorpora el DAP y la ALCO, así: VOLCO $=\left[(\mathrm{DAP} / 100)^{2 *} 0,7854 * A L C O * 0,70\right]$. La

Tabla 1. Localización (municipios) de las plantaciones donde se realizó la selección de árboles plus de Acacia mangium en el departamento de Córdoba, Colombia.

\begin{tabular}{|c|c|c|c|c|}
\hline \multirow{2}{*}{ Municipio } & \multirow{2}{*}{ Plantaciones } & \multirow{2}{*}{$\begin{array}{l}\text { Área } \\
\text { (has) }\end{array}$} & \multicolumn{2}{|c|}{ Coordenadas } \\
\hline & & & Latitud & Longitud \\
\hline \multirow{3}{*}{ TIERRALTA } & Urrá & 2300 & $08^{\circ} 02^{\prime} 05,5^{\prime \prime}$ & $076^{\circ} 12^{\prime} 01,2^{\prime \prime}$ \\
\hline & Halicarnaso & 240 & $08^{\circ} 09^{\prime} 06,6^{\prime \prime}$ & $075^{\circ} 57^{\prime} 48,0^{\prime \prime}$ \\
\hline & La Dinastía & 245 & $08^{\circ} 08^{\prime} 33,5^{\prime \prime}$ & $075^{\circ} 57^{\prime} 28,2^{\prime \prime}$ \\
\hline \multirow{2}{*}{ PLANETA RICA } & Cucharita & 220 & \multirow{2}{*}{$08^{\circ} 24^{\prime} 47,6^{\prime \prime}$} & \multirow{2}{*}{$075^{\circ} 36^{\prime} 16,9^{\prime \prime}$} \\
\hline & La Independencia & 150 & & \\
\hline AYAPEL & Santiago de Compostela & 110 & $08^{0} 12^{\prime} 33,0^{\prime \prime}$ & $075^{\circ} 05^{\prime} 07,6^{\prime \prime}$ \\
\hline MONTERÍA & La Lorena & 350 & $08^{\circ} 29^{\prime} 42,7^{\prime \prime}$ & $075^{\circ} 46^{\prime} 23,6^{\prime \prime}$ \\
\hline PUERTO LIBERTADOR & El Taller & 10 & $07^{0} 51^{\prime} 33,2^{\prime \prime}$ & $075^{\circ} 43^{\prime 22,4 "}$ \\
\hline SAN ANTERO & Refopal & 1 & $09^{\circ} 18^{\prime} 26,6^{\prime \prime}$ & $075^{\circ} 50^{\prime} 20,6^{\prime \prime}$ \\
\hline Total & & 3.626 & & \\
\hline
\end{tabular}

CALI, a través de la siguiente ecuación: CALI $=[($ FUSTE*0,7) $+($ RAMA*0,2) + (SANIDAD*0,1)].

Los árboles seleccionados, se clasificaron en dos listas A y B. En A, se incluyeron los árboles que registraron superioridad con sus mejores vecinos, tanto en VOLCO como en CALI, con base en el diferencial de selección:

$$
\left.S=\left[\left(Y_{\text {árbol seleccionado }}-\tilde{Y}_{\text {árboles vecinos }}\right) / \tilde{Y}_{\text {árboles vecinos }}\right) * 100\right] \text {; }
$$

mientras que $\mathrm{B}$, comprendió los árboles plus que superaron solamente en VOLCO o en CALI, a sus mejores vecinos o comparadores. El carácter calidad, se transformó a una escala de 1 a 100, para facilitar su comprensión e interpretación, así: $\mathrm{CALI}_{\text {Invertida }}=\{1-[(\mathrm{CALI}-1) / 3]\}$. Con los valores obtenidos del diferencial de selección S, se estimó el valor promedio de S: i) por cada plantación; ii) para todos los árboles seleccionados y, iii) para únicamente los árboles seleccionados en la lista $\mathrm{A}$ (Zobel E Talbert, 1984; Murillo et al. 2004).

La ganancia genética (GG), se valoró a través de la siguiente ecuación (Zobel E Talbert, 1984; Murillo et al. 2004): GG $=\mathrm{S}^{*} \mathrm{~h}^{2}$, donde, "S" es el diferencial de selección y $\mathrm{h}^{2}$ es la heredabilidad en sentido estrecho promedio, reportada para varias especies, incluida la $A$. mangium: $\mathrm{h}^{2}$ Diámetro $=0,20$; $\mathrm{h}_{\text {Altura }}^{2}=0,25 ; \mathrm{h}_{\text {Volumen }}=0,25 \mathrm{y} \mathrm{h}^{2}$ Calidad $=0,40$ (Cornelius, 1994).

Con la GG obtenida, se determinó un Índice de Selección (IS), que integró, de forma ponderada, el VOLCO con la CALI, así (Murillo et al. 2004):

IS $=[(0,6 * \mathrm{VOLCO} / \mathrm{ds})+(0,4 * \mathrm{CALI} / \mathrm{ds})]$, en donde "ds" es la desviación estándar de cada carácter. 
Con base en el "IS", se logró el ranking de los mejores 15 árboles seleccionados, los cuales, pueden constituir la población comercial y, el resto de los árboles, se convertirán en la población de mejoramiento e investigación. Todos los cálculos estadísticos fueron realizados con ayuda de la hoja electrónica EXCEL.

\section{RESULTADOS Y DISCUSIÓN}

Árboles plus seleccionados e intensidad de selección: En la tabla 2, se puede observar que el proceso de selección, adelantado en las 3.626 hectáreas muestreadas, permitió identificar un total de 89 árboles plus, por sus características fenotípicas sobresalientes. Sólo cinco plantaciones, de nueve muestreados, permitieron identificar árboles superiores. El número de árboles seleccionados por plantación osciló entre 2 y 52 y, esta variación, se debe, fundamentalmente, a las diferencias en tamaño de las plantaciones, las cuales, fluctuaron entre 1 y 2.300 hectáreas (Tabla 1). Otro factor que pudo afectar la cantidad de árboles seleccionados por lote, puede ser la variabilidad y el origen genético de la semilla sexual utilizado para la siembra de las plantaciones, objeto de estudio, que se pudo detectar en la visita de selección y de eliminación de árboles en las plantaciones, hasta el punto que en cuatro de ellas (La Dinastía, La Independencia, La Lorena y Refopal), no se incluyó ningún árbol superior.
Con base en el proceso de clasificación anterior, se obtuvo una intensidad de selección por plantación, que osciló desde 1, por cada 3.459 árboles (Urrá), a 1, por cada 30.553 árboles (Cucharita), lo que originó una intensidad de selección promedio de un árbol seleccionado por cada 10.622 evaluados (Tabla 2). Esta intensidad de selección promedia resultó ser un poco mayor, que la reportada por Vallejos et al. (2010) en GENFORES (Costa Rica), de un árbol plus por cada 15.000 a 20.000 individuos.

En la misma tabla 2, se puede observar que de los 89 árboles escogidos, el 36\%, aproximadamente (32), superó a sus mejores vecinos en los dos criterios: volumen y calidad (Plus A); mientras que el $64 \%$ restante de los árboles seleccionados (57), sólo superaron a sus vecinos en volumen o en calidad (Plus B). El número de árboles plus A por plantación fluctuó entre 1 y 17. La intensidad de selección para este grupo de árboles fue mucho mayor: entre 1, de cada 8.888 árboles (Reforestadora del Caribe), a 1, por cada 61.105 evaluados (Cucharita), que en promedio originó, una intensidad de selección de un árbol seleccionado, por cada 30.538. Esta intensidad de selección, promedio para este grupo de árboles, resultó ser más exigente que la obtenida por Murillo \& Badilla (2009), quienes reportaron intensidades de un árbol plus, por cada 15.000 a 20.000 individuos e, igualmente, superior a las intensidades recomendadas por Zobel \& Talbert (1984), quienes sugieren

Tabla 2. Árboles seleccionados e intensidad de selección por plantación en las poblaciones estudiadas de Acacia mangium, en el departamento de Córdoba, Colombia.

\begin{tabular}{|c|c|c|c|}
\hline \multirow{2}{*}{ Municipio } & Plantación & $\begin{array}{c}\text { Árboles Plus } \\
\text { seleccionados }\end{array}$ & $\begin{array}{c}\text { Intensidad de Selección } \\
\text { (árboles) }\end{array}$ \\
\hline \multirow{2}{*}{ Tierralta } & Urrá & 18 & 1 de 3.459 \\
\cline { 2 - 5 } & Halicarnaso & 52 & 1 de 9.978 \\
\hline Planeta Rica & Cucharita & 7 & 1 de 30.553 \\
\hline Ayapel & Santiago de Compostela & 10 & 1 de 12.221 \\
\hline Puerto Libertador & Reforestadora del Caribe & 2 & 1 de 4.444 \\
\hline \multicolumn{2}{|c|}{ TOTAL } & 89 & 1 de 10.622 \\
\hline \multirow{2}{*}{ Municipio } & Plantación & Árboles Plus A & Intensidad de Selección \\
& Uárboles) \\
\hline \multirow{2}{*}{ Tierralta } & Urrá & 5 & 1 de 12.576 \\
\hline Planeta Rica & Halicarnaso & 17 & 1 de 31.696 \\
\hline Ayapel & Cucharita & 4 & 1 de 61.105 \\
\hline Puerto Libertador & Santiago de Compostela & 5 & 1 de 24.442 \\
\hline \multicolumn{2}{|c|}{ Reforestadora del Caribe } & 1 & 1 de 8.888 \\
\hline \multicolumn{2}{|c|}{ TOTAL } & 32 & 1 de 30.538 \\
\hline
\end{tabular}


intensidades de selección de 1: $<20$, para rodales semilleros y 1: $>1.000$, para huertos semilleros. Los valores de intensidad de selección obtenidos en este estudio, se consideran excelentes para programas de mejoramiento genético que se inician, ya que además de permitir identificar un número importante de árboles superiores, también hace posible racionalizar y hacer eficiente el proceso de mejoramiento.

Diferencial de selección y ganancia genética esperada. En la tabla 3, se detalla que el diferencial de selección promedio (\%) varió con el tipo de plantación y los cuatro caracteres evaluados. El origen del material (aspecto genético) y las condiciones de manejo de las plantaciones (aspecto ambiental) influyen, significativamente, en la posibilidad de localizar un número mayor de árboles plus. Es de aclarar, que a pesar que el método de selección reduce, de forma considerada, el error de incluir árboles que no sean superiores genéticamente, éste sigue siendo un método de evaluación fenotípica, que no logra filtrar todos estos casos; por tanto, todo programa de mejoramiento genético requiere, de manera obligada, una siguiente fase de evaluación o de comprobación genética en campo.

Las plantaciones que permitieron lograr los mayores diferenciales de selección en volumen comercial (VOLCO) y calidad del fuste (CALI), fueron: Reforestadora del Caribe (111,0\% y $65,4 \%$ ) y Urrá (143,6\% y 60,1\%). En su orden, los caracteres donde los 89 árboles escogidos presentaron el mayor diferencial de selección, fueron: volumen comercial por árbol (VOLCO), altura comercial (ALCO), calidad del fuste (CALI) y diámetro a la altura del pecho (DAP), con valores promedios de superioridad de 118,1; 70,6; 48,6 y $10,3 \%$, respectivamente. Estos valores son superiores a los reportados en melina por Kumar $\&$ Matharoo (2003), quienes han encontrado diferenciales de selección en un $40 \%$, para altura total; $106 \%$, para altura comercial y $66 \%$, en DAP, con respecto a la población original.

A pesar de lo anterior, se debe tener presente, como lo señalan Vallejos et al. (2010), que estos valores de diferencial de selección son, por lo general, inferiores a los valores reales, debido a que cada árbol plus fue evaluado contra sus mejores cuatro vecinos. Se debe recordar que los cuatro mejores vecinos son los mejores competidores en el mismo micrositio (mismo ambiente) y, por tanto, el verdadero diferencial de selección, se puede pensar que será ligeramente superior al reportado con el método de selección.

La misma tendencia en el diferencial de selección, se puede advertir con los cinco caracteres evaluados, cuando sólo se consideran los 32 árboles plus A. Los mayores diferenciales de selección, se obtuvieron en: VOLCO (194,3\%), ALCO (89,0\%), CALI $(51,7 \%)$ y DAP $(22,4)$. Si se analiza solamente los 32 árboles plus de la lista $\mathrm{A}$ (del total de los 89 árboles plus de las listas $A+B$ ), se puede distinguir un potencial mayor de mejoramiento genético en VOLCO, de 76,2 puntos más en porcentaje y, en ALCO, de 18,4 puntos más en porcentaje. Los valores de diferencial de selección registrados en este estudio son mayores a los reportados por Vallejos et al. (2010) en teca, en Costa Rica, de $22,88 \%$, en volumen y $21,83 \%$, en calidad y de $35,49 \%$ (volumen) y $33,7 \%$ (calidad), para los árboles de la lista $\mathrm{A}$, respectivamente.

Tabla 3. Diferencial de selección promedio de los árboles plus de Acacia mangium, seleccionados en el departamento de Córdoba, Colombia*.

\begin{tabular}{|c|c|c|c|c|c|}
\hline PLANTACIÓN & $\begin{array}{c}\text { Árboles } \\
\text { Seleccionados }\end{array}$ & $\begin{array}{c}\text { DAP } \\
(\%)\end{array}$ & $\begin{array}{c}\text { ALCO } \\
(\%)\end{array}$ & $\begin{array}{c}\text { VOLCO } \\
(\%)\end{array}$ & $\begin{array}{c}\text { CALI } \\
(\%)\end{array}$ \\
\hline $\begin{array}{c}\text { Puerto Libertador } \\
\text { (Reforestadora del Caribe) }\end{array}$ & 2 & 10,2 & 76,2 & 111,0 & 65,4 \\
\hline $\begin{array}{c}\text { Ayapel } \\
\text { (Santiago de Compostela) }\end{array}$ & 10 & 14,6 & 33,3 & 102,6 & 57,2 \\
\hline Planeta Rica (Cucharita) & 7 & 10,6 & 54,9 & 104,7 & 42,5 \\
\hline Tierralta (Halicarnaso) & 52 & 11,2 & 65,4 & 114,4 & 43,1 \\
\hline Tierralta (Urrá) & 18 & 5,1 & 111,0 & 143,6 & 60,1 \\
\hline Global & 89 & 10,3 & 70,6 & 118,1 & 48,6 \\
\hline Plus A & 32 & 22,4 & 89,0 & 194,3 & 51,7 \\
\hline
\end{tabular}

*Diámetro a la altura del pecho (DAP), Altura comercial (ALCO), Volumen comercial (VOLCO) y Calidad del fuste (CALI) en una escala de 0 a 100. 
La tabla 4 indica el resultado en diferencial de selección y ganancia genética que se obtendría al seleccionar los mejores 15 árboles plus. En este caso, se logran incrementos importantes en el diferencial de selección, respecto a los 32 árboles plus $\mathrm{A}$, especialmente, en VOLCO (52,7 puntos más en porcentaje), ALCO (30,0 puntos más en porcentaje) y CALI (16,5 puntos más en porcentaje). Más no así en DAP (-0,5 puntos menos en porcentajes), como suele ocurrir, debido al alto efecto ambiental, para este carácter (Murillo et al. 2004). Estos valores son superiores en mayor proporción a los reportados en melina, por Kumar $\&$ Matharoo (2003) y, en teca, por Vallejos et al. (2010), en Costa Rica. Los resultados de este estudio justifican el riguroso proceso de selección realizado en las plantaciones evaluadas y permiten suponer un importante progreso genético.

Si se seleccionan únicamente los mejores 15 árboles plus de la lista A, se podría estimar una ganancia genética esperada de $64,93 \%$, en VOLCO; $31,08 \%$, en ALCO; $26,64 \%$, en CALI y $4,57 \%$, en DAP. Estos valores de ganancia genética son superiores a los que se conseguirían sí se utilizaran todos los 32 árboles plus de la lista A (Tabla 4). Los estimados registrados son mayores a los reportados por varios autores: a) Vallejos et al. (2010), quienes relacionan ganancias genéticas esperadas, en general, entre 20 - 25\% en volumen; b) Mesén (2001), quien estimó ganancias genéticas en melina, de 17\%, en altura y 43\%, en DAP; c) Cornelius \& Hernández (1994), en la misma especie, reportaron ganancias genéticas de hasta $12 \%$, en rectitud del fuste; d) Kumar $E$ Matharoo (2003), en melina, a nivel clonal, encontraron para altura, diámetro basal y diámetro a la altura del pecho, ganancias de un 18\%, 25\% y $30 \%$, respectivamente; e) Rojas \& Arias (2004), en Pinus caribaea var. hondurensis Barr., en la zona del Pacífico sur de Costa Rica, con ganancias del $23 \%$, en volumen.

Si se tiene en cuenta que, tradicionalmente, en la mayoría de los programas de mejoramiento genético, con incrementos del $4 \%$ por conceptos de ganancias genéticas en volumen se recupera la inversión realizada (Ipinza, 1998; Cornelius \& Ugarte-Guerra, 2010), los resultados registrados en este estudio, permiten deducir alta rentabilidad económica en el programa que se adelanta en Córdoba.

En la tabla 5, se presenta la relación y el código de los 15 mejores árboles plus, dentro de la lista $\mathrm{A}$, con sus valores de diferencial de selección y clasificados, de acuerdo a su índice de selección (IS). Se corrobora lo detectado, anteriormente, para diferencial de selección, que los caracteres en los cuales se localizó la mayor ganancia genética esperada, son en su orden: VOLCO, ALCO, CALI y DAP.

Los 32 árboles plus de la lista A, por sus características superiores, pueden ser utilizados en el corto plazo, como progenitores para siembras comerciales (población comercial), empleando su semilla sexual (aprovechamiento de la recombinación y sólo de la varianza genética aditiva) o clonándolos directamente (aprovechamiento de toda la varianza genética y mantenimiento del genoma deseado), para lograr la mayor ganancia genética (Ipinza, 1998; Mesén, 2001; Murillo \& Badilla, 2009; Vallejos et al. 2010); sin embargo, se puede aumentar aún más la ganancia genética esperada, utilizando para el mismo propósito, los mejores 15 árboles

Tabla 4. Diferencial de selección (S) porcentual (\%) y ganancia genética (GG) esperada por grupo de árboles seleccionados de Acacia mangium, en el departamento de Córdoba, Colombia.

\begin{tabular}{|c|c|c|c|c|}
\hline Grupo de árboles & DAP & ALCO & VOLCO & CALI \\
\hline S global de 89 árboles (\%) & 10,3 & 70,6 & 118,1 & 48,6 \\
\hline S de 32 árboles plus A (\%) & 22,4 & 89,0 & 194,3 & 51,7 \\
\hline S de 15 árboles plus A (\%) & 21,9 & 119,0 & 247,0 & 68,2 \\
\hline GG esperada Global de 89 árboles (\%) & 2,06 & 17,64 & 29,53 & 19,44 \\
\hline GG esperada de 32 árboles plus A (\%) & 4,48 & 22,24 & 48,57 & 20,67 \\
\hline GG esperada de 15 árboles plus A (\%) & 4,57 & 31,08 & 64,93 & 26,64 \\
\hline
\end{tabular}

*Diámetro a la altura del pecho (DAP), Altura comercial (ALCO), Volumen comercial (VOLCO) y Calidad del fuste (CALI) en una escala de 0 a 100. 
Tabla 5. Ranking del diferencial de selección (S) del índice de selección (IS) y ganancia genética esperada (GG), para los 15 mejores árboles plus, seleccionados de Acacia mangium, en el departamento de Córdoba, Colombia*.

\begin{tabular}{|c|c|c|c|c|c|c|}
\hline Clasificación & Código árbol & $\begin{array}{c}S \\
\text { DAP }\end{array}$ & $\begin{array}{c}S \\
\text { ALCO }\end{array}$ & $\begin{array}{c}\text { S } \\
\text { VOLCO }\end{array}$ & $\begin{array}{c}S \\
\text { CALI }\end{array}$ & IS \\
\hline 1 & 21112 & 28,7 & 256,3 & 558,7 & 56,3 & 3,6 \\
\hline 2 & 41004 & 79,6 & 123,2 & 620,4 & 36,6 & 3,4 \\
\hline 3 & 11024 & 20,6 & 233,3 & 401,7 & 64,3 & 3,0 \\
\hline 4 & 21089 & 19,3 & 89,6 & 174,1 & 108,9 & 2,9 \\
\hline 5 & 11012 & 27,5 & 200,0 & 385,9 & 47,2 & 2,6 \\
\hline 6 & 11023 & 11,3 & 147,9 & 218,4 & 78,0 & 2,5 \\
\hline 7 & 21067 & 17,2 & 177,5 & 284,6 & 62,3 & 2,5 \\
\hline 8 & 11010 & 8,1 & 64,6 & 94,0 & 86,6 & 2,1 \\
\hline 9 & 31029 & 8,6 & 45,2 & 68,9 & 90,4 & 2,1 \\
\hline 10 & 31005 & 19,0 & 118,8 & 221,4 & 52,7 & 2,0 \\
\hline 11 & 31008 & 36,9 & 63,3 & 207,0 & 52,7 & 1,9 \\
\hline 12 & 21053 & 26,9 & 80,8 & 195,3 & 52,7 & 1,9 \\
\hline 13 & 31028 & 7,5 & 59,8 & 91,4 & 75,0 & 1,9 \\
\hline 14 & 21071 & 8,6 & 80,0 & 114,2 & 68,8 & 1,8 \\
\hline 15 & 21084 & 33,9 & 73,6 & 226,0 & 40,6 & 1,8 \\
\hline \multicolumn{2}{|c|}{ Promedio } & 22,85 & 124,31 & 259,72 & 66,59 & \\
\hline \multicolumn{2}{|c|}{ Varianza } & 333,42 & 4542,91 & 27713,67 & 398,54 & \\
\hline \multicolumn{2}{|c|}{ Error estándar } & 4,88 & 18,01 & 44,49 & 5,34 & \\
\hline \multicolumn{2}{|c|}{ GG esperada (\%) } & 4,57 & 31,08 & 64,93 & 26,64 & \\
\hline
\end{tabular}

*Diámetro a la Altura del Pecho (DAP), Altura Comercial (ALCO), Volumen Comercial (VOLCO), Calidad del Fuste (CALI), en una escala de 0 a 100, GG = ganancia genética e IS = Índice de selección.

plus, de la lista A. Los árboles plus B (57 restantes), no se incorporarán a la población comercial, dado que representan una condición de superioridad solamente en uno de los dos caracteres: volumen ó calidad, por lo tanto, formarán parte de la población de mejoramiento (investigación y desarrollo) y se mantendrán a la espera de su evaluación genética y su manejo en los cruzamientos controlados en el programa de mejoramiento de acacia en Córdoba, como lo señalan Murillo E Badilla (2009) y Vallejos et al. (2010).

Estos resultados le permiten, igualmente, al mejorador forestal, de acuerdo a sus recursos económicos y disponibilidad de sitios, definir si incluye en sus ensayos de progenies todos los 89 árboles plus seleccionados, o los 32 árboles plus de la lista $\mathrm{A}$, o los mejores 15 árboles, de la lista A. Como estrategia de mejoramiento, se debe buscar establecer los ensayos genéticos en, al menos, tres ambientes diferentes y representativos de las mayores unidades edafo-climáticas existentes en la región. Estos ensayos permitirían, después del raleo genético, su conversión a sendos huertos semilleros de primera generación, que ofrecerían semilla de excelente calidad genética, para nuevas siembras comerciales. Ello, a la vez, facilita contar con material vegetal para clonar los mejores árboles de las óptimas familias, con adaptación específica en cada ambiente. Esta estrategia haría posible que los árboles definitivos se crucen entre sí, forzando su recombinación genética, la acumulación de alelos favorables y, por consiguiente, la producción de semilla de calidad genética superior, para continuar con el proceso de mejoramiento (Ipinza, 1998; Mesén, 2001).

Como es de esperarse, es posible que se encuentre mejor material genético en otras empresas, organizaciones o países; por consiguiente este grupo de árboles plus A, que 
se ha aislado y con el que se da inicio a un programa de mejoramiento genético de acacia en Córdoba, como lo señalan Murillo \& Badilla (2009), permite, igualmente, el desarrollo de modelos cooperativos o de alianza e intercambio de material genético con otras compañías. Ello a la vez, junto con la introducción de germoplasma de procedencias fuera del país, hace posible reducir costos en el programa de mejoramiento, generar mayores ganancias genéticas y ampliar la base genética del programa, con lo que se garantizaría que la población de mejoramiento no estará altamente emparentada al cabo de tres generaciones de mejoramiento genético. La población comercial (los mejores 15 materiales) podría, entonces, continuar ofreciendo altos diferenciales de selección y ganancia genética realizable.

Los resultados obtenidos sugieren un progreso genético significativo en el mejoramiento de Acacia mangium en Córdoba. De acuerdo con Xavier et al. (2009) y Zobel E Talbert, (1984), este avance integrado con el proceso de silvicultura clonal con los mejores árboles élites en la zonas productoras, constituirían el complemento ideal del programa de mejoramiento genético de acacia. Adicionalmente, hacen prever un aporte importante a la productividad, competitividad y sostenibilidad de la producción forestal en el departamento, de acuerdo a las exigencias de calidad del mercado internacional, al cual, se pretende llegar. No obstante, es necesario corroborar este gran progreso genético, mediante ensayos de progenies en varias zonas productoras de Córdoba.

Agradecimientos. Al Ministerio de Agricultura y Desarrollo Rural de Colombia, Universidad de Córdoba, Cadena Forestal de Córdoba, Instituto Tecnológico de Costa Rica (ITCR) y 3F Kanguroid, por la cofinanciación y el apoyo logístico para la realización de la investigación. Conflictos de intereses. El artículo fue preparado y revisado con la participación de todos los autores, quienes declaramos que no existe ningún conflicto de intereses que ponga en riesgo la validez de los resultados presentados. Financiación. Este estudio fue financiado por el Ministerio de Agricultura y Desarrollo Rural de Colombia, Universidad de Córdoba, Cadena Forestal de Córdoba y la empresa 3F Kanguroid, a través del proyecto "Selección de árboles plus y creación de fuentes semilleras de teca (Tectona grandis), melina (Gmelina arborea Roxb) y acacia (Acacia mangium Willd.) en el departamento de Córdoba”.

\section{BIBLIOGRAFÍA}

1. BALCORTA, H.; VARGAS, J. 2004. Variación fenotípica y selección de árboles en una plantación de melina (Gmelina arborea Linn., Roxb.) de tres años de edad. Revista Chapingo (México), Serie ciencias forestales y del ambiente. 10(1):13-19.
2. BLADA, I.; POPESCU, F. 2008. Diallel crossing in Pinus cembra: IV. Age trends in genetic parameters and genetic gain for growth and branching traits. Ann. For. Res (Rumania). 51:89-112.

3. BOTREL, M.C.G.; MOREIRA DA SILVA, J.R.; TRUGILHO, P.F.; DA SILVA, S.C.; BRUNO RICARDO, B.F. 2007. Ganho genético em propiedades físicas e mecânicas de clones de eucalipto. Sci. For., Piracicaba (Brasil). 76:13-19.

4. CATIE. 1992. Proyecto de Diseminación del Cultivo de Árboles de Uso Múltiple en América Central y Panamá. Acacia mangium Willd.; Especie de Árbol de Uso Múltiple en América Central. Centro Agron. Tropical de Investigación y Enseñanza, Turrialba, Costa Rica. 57p.

5. CFC-CADENA FORESTAL DE CÓRDOBA. 2000. Acuerdo Regional de Competitividad para la Cadena Forestal en el Departamento de Córdoba (Colombia). 14p. Disponible desde internet en http://www.conif.org.co/docs/ acuerdo_reg_cordoba.doc (con acceso 23/12/09).

6. CORNELIUS, J.; UGARTE-GUERRA, L. 2010. Introducción a la Genética y domesticación forestal para la Agroforestería y Silvicultura. Notas de clase. Lima, Perú. Centro Mundial para la Agroforestería (ICRAF). 124p.

7. CORNELIUS, J. 1994. The effectiveness of plus tree selection for yield. Forest Ecology and Management (USA). (67):23-34.

8. CORNELIUS, J.; HERNÁNDEZ, M. 1994. Variación genética en crecimiento y rectitud del fuste en Gmelina arborea en Costa Rica. Bol. Mejoramiento Genético y Semillas Forestales. 10:9-12.

9. HAN, S.U.; KANG, K.S.; CHEON, B.H.; KIM, C.S. 2007. Realized genetic gains and heritabilities for height, $\mathrm{DBH}$ and volume growth in open-pollinated progenies of Pinus thunbergii. Korean J. Breeding Science. 39(1):15-19.

10. IPINZA, R. 1998. Mejoramiento genético forestal. Serie Técnica N42, Programa CONIF-Ministerio de Agricultura sobre investigaciones en semillas de especies forestales nativas. INSEFOR. Santa Fe de Bogotá, Colombia. 162p.

11. KUMAR, A.; MATHAROO, A.K. 2003. Genetic improvement of Gmelina arborea in India. In: Recent Advances with Gmelina arborea (eds. W. S. Dvorak, G. R. Hodge, W. C. Woodbridge and J. L. Romero). North Carolina State University. Raleigh, NC. USA. CAMCORE. 221p. 
12. LADRACH, W. 2010. Expansion of pulp production in the third world. Disponible desde internet en http:// www.alleghenysaf.org/winter_2010.htm (con acceso 26/02/10).

\section{MADR-MINISTERIO DE AGRICULTURA Y DESARROLLO} RURAL. 2005. Características y estructura del sector forestal-madera-muebles en Colombia, una mirada global de su estructura y dinámica 1991-2005. Documento de trabajo No. 95. Ministerio de Agricultura y Desarrollo Rural. Observatorio Agrocadenas Colombia. 63p.

14. MESÉN, F. 2001. Introducción al mejoramiento genético forestal. En: Identificación, selección y manejo de fuentes semilleras. Serie Técnica / No. 32. Convenio CONIF, INSEFOR y MADR. Bogotá (Colombia), septiembre. ISSN 0121-0300. 118p.

15. MURILLO, O.; BADILLA, Y. 2004. Evaluación de la calidad y estimación del valor en pie de la plantación forestal. Escuela de Ingeniería Forestal, ITCR. Cartago, Costa Rica. 50p.

16. MURILLO, O.; BADILLA, Y. 2009. Reproducción clonal de árboles. Escuela de Ingeniería Forestal, ITCR. Cartago, Costa Rica. 45p.

17. MURILLO, O.; BADILLA, Y. 2005. ¿Qué es mejoramiento genético forestal? Manual. Taller de Publicaciones del Instituto Tecnológico de Costa Rica. Escuela de Ing. Forestal. Cartago, Costa Rica. 14p.

18. MURILLO, O.; OBANDO, G.; BADILLA, Y.; ARAYA, E. 2004. GENFORES, a Costa Rican tree improvement and gene conservation cooperative. En: IUFRO Meeting. Forest Genetics and Genomics. November 1-5. Charleston, South Carolina, USA.

19. RINCÓN, M. 2009. El sector forestal en Córdoba: Cadena productiva forestal madera y muebles departamento de Córdoba. Informe Cadena Forestal de Córdoba (Colombia), febrero de 2009 (Centro de Investigaciones Turipaná - Corpoica). 37p.
20. ROCHA, R.B.; VIEIRA, A.H.; BENTES, M.D.; BRUM, L.M. 2009. Avaliação genética de procedências de bandarra (Schizolobium amazonicum) utilizando REML/BLUP (Máxima verossimilhança restrita/Melhor predição linear não viciada. Sci. For., Piracicaba (Brasil), 37(84): 351-358.

21. ROJAS, F;; ARIAS, D. 2004. Manual para productores de Melina (Gmelina arborea) en Costa Rica. Cartago (Costa Rica). 86p.

22. TRUJILLO, E. 2007. Guía de reforestación. Primera edición. El semillero y PROFAFOR del Ecuador S.A., Bogotá D.C (Colombia). 178p.

23. VALLEJOS, J.; BADILLA, Y.; PICADO, F.; MURILLO, O. 2010. Selección e incorporación de árboles plus en programas de mejoramiento genético forestal. Agr. Costarricense (Costa Rica). 34(1) (en prensa).

24. VERRYN, S.D.; SNEDDEN, C.L.; EATWELL, K.A. 2009. A comparison of deterministically predicted genetic gains with those realized in a South African Eucalyptus grandis breeding program. J. Forest Science, Menlo Park (Czech Republic). 71(2):141-146.

25. XAVIER, A.; WENDLING, I.; DA SILVA, R. 2009. Silvicultura Clonal. Princípios e Técnicas. Editorial Universidad Federal de Viçosa. Viçosa, Minas Gerais, Brasil. 272p.

26. ZOBEL, B.; TALBERT, J. 1984. Applied Forest Tree Improvement. John Wiley \& Sons. New York, USA. 510p.

27. ZOBEL, B.; TALBERT, J. 1988. Técnicas de mejoramiento genético de árboles forestales. Ed. Limusa. México D.F. 545p.

Recibido: Abril 27 de 2010

Aceptado: Octubre 18 de 2010 